\title{
Direct determination of the generalized Stokes parameters from the usual Stokes parameters
}

\author{
Bhaskar Kanseri, ${ }^{1,2, *}$ Shyama Rath, ${ }^{2}$ and Hem Chandra Kandpal ${ }^{1}$ \\ ${ }^{1}$ Optical Radiation Standards, National Physical Laboratory, New Delhi 110012, India \\ ${ }^{2}$ Department of Physics and Astrophysics, University of Delhi, Delhi 110007, India \\ *Corresponding author: kanserib@mail.nplindia.ernet.in
}

Received December 1, 2008; revised January 21, 2009; accepted January 23, 2009; posted February 3, 2009 (Doc. ID 104672); published March 3, 2009

\begin{abstract}
We report an experimental method to determine the generalized Stokes parameters for a pair of points in the cross section of an electromagnetic beam, e.g., an expanded laser beam, with the help of a Young's interferometer and a set of polarizers and quarter-wave plates. The method is investigated theoretically using the electromagnetic spectral interference law. The generalized Stokes parameters, owing to their two-point nature, determine the behavior of the single-point polarization properties of the electromagnetic beam at a field point. The present method offers a unique means to determine the two-point parameters (correlation functions) by measuring the usual Stokes parameters (intensities) and the contrast parameters (visibilities) of the beam. The method might be applicable to determine the polarization dependent changes in various optical measurements. (C) 2009 Optical Society of America

OCIS codes: $260.3160,030.1640$.
\end{abstract}

Recently proposed generalized Stokes parameters [1], treated as a two-point extension of the usual Stokes parameters $[2,3]$, play an important role in determining the change in polarization properties of random electromagnetic beams on propagation $[4,5]$. These parameters may be written in terms of the correlations between electric field components at a pair of points, which equip them to characterize simultaneously the coherence and the polarization properties of the electromagnetic fields. These complex quantities conform to the unified theory of coherence and polarization [6,7], providing an excellent way for polarization modulation studies [8-10]. Not long ago it was shown theoretically that the usual Stokes parameters at an observation plane not only are the sum of corresponding Stokes parameters produced by two pinholes separately, but also are modulated by the generalized Stokes parameters on the pinholes. In other words, changes in one-point correlation properties (usual Stokes parameters) of the electromagnetic beam on propagation are governed by the two-point correlation properties (generalized Stokes parameters) of the beam [11]. This relationship is termed as the electromagnetic spectral interference law [9].

The usual Stokes parameters were determined experimentally by placing a polarizer and a quarterwave plate in the beam, with appropriate directions of polarizations [2]. In the recent past, we presented a method [12] in which the generalized Stokes parameters were determined from the cross-spectral density matrix using a set of polarizers and rotators in a modified version of Young's interferometer. In this method, for the sake of simplicity, the spectral measurements were taken at an axial point on the observation plane, where the fringe intensity was maximum. This reduced the complex parameters into real ones. The method is quite useful in laboratory scale; however, practical situations are otherwise, i.e., either the observation point is an off-axis point or the light is partially coherent. For partially coherent light, it is quite complex to use the modified assembly [12] consisting of various mirrors and prisms in the conventional Young's interferometer due to little coherence and low-light-level considerations. Also keeping in view the roles of the two kinds of Stokes parameters in characterizing the polarization properties of the beam, it is important to determine the generalized Stokes parameters directly from the usual Stokes parameters.

In this Letter, we present a simple and unique method to determine the generalized Stokes parameters for a pair of points in the cross section of an expanded laser beam. Making use of the electromagnetic spectral interference law mathematical expressions for generalized Stokes parameters are derived. With the help of these expressions the generalized Stokes parameters for a pair of points in the cross section of an expanded laser beam are determined experimentally using the usual Stokes parameters and visibility measurements. The single-point Stokes parameters are determined experimentally by using a pair of optical elements only with the conventional Young's interferometer. We have taken a general case in which the observation point is an off-axis point and spectral measurements were taken in and around that point. The laser beam was chosen owing to its wide optical applications.

The usual Stokes parameters $S_{n}(\mathbf{r}, \omega), n=0,1,2,3$ due to both the beams at point $\mathrm{P}$ in plane $\mathrm{R}$ (Fig. 1) are expressed in terms of the Stokes parameters due to individual beams $S_{n}^{(i)}(\mathbf{r}, \omega), i=1,2$ by the electromagnetic spectral interference law [9],

$$
\begin{aligned}
S_{n}(\mathbf{r}, \omega)= & S_{n}^{(1)}(\mathbf{r}, \omega)+S_{n}^{(2)}(\mathbf{r}, \omega)+2 \sqrt{S_{0}^{(1)}(\mathbf{r}, \omega) S_{0}^{(2)}(\mathbf{r}, \omega)} \\
& \times\left|\mu_{n}\left(\mathbf{r}_{1}, \mathbf{r}_{2}, \omega\right)\right| \cos \beta_{n},
\end{aligned}
$$

where $\beta_{n}, \quad(n=0,1,2,3)$ is the phase term of $\mu_{n}\left(\mathbf{r}_{1}, \mathbf{r}_{2}, \omega\right)$ and $-1 \leqslant \cos \beta_{n} \leqslant 1$. The absolute values of $\mu_{n}\left(\mathbf{r}_{1}, \mathbf{r}_{2}, \omega\right)$ are the modulation contrasts $C_{n}(\mathbf{r}, \omega)$ 


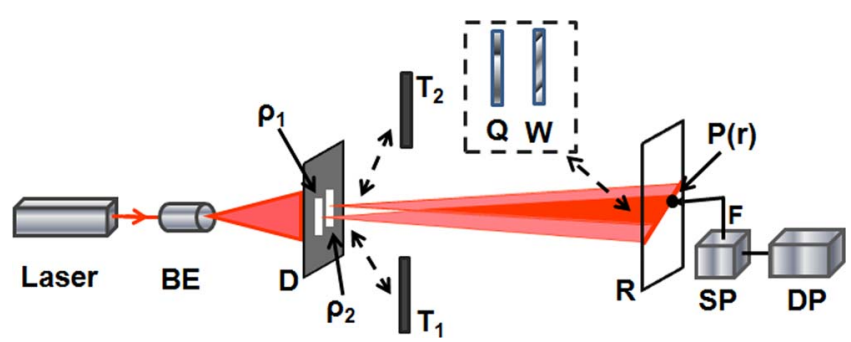

Fig. 1. (Color online) Schematics of the experimental arrangement. $\mathrm{BE}$ is a beam expander, $\mathrm{T}_{1}$ and $\mathrm{T}_{2}$ are stops, $\mathrm{Q}$ is a polarizer, and $\mathrm{W}$ is a quarter-wave plate. SP is a spectrometer coupled with a fiber $\mathrm{F}$ and $\mathrm{DP}$ is a data processor. Other notations have their usual meaning.

associated with the Stokes parameters and are given by [9]

$$
\left|\mu_{n}\left(\mathbf{r}_{1}, \mathbf{r}_{2}, \omega\right)\right|=\frac{S_{n}^{\max }(\mathbf{r}, \omega)-S_{n}^{\min }(\mathbf{r}, \omega)}{S_{0}^{\max }(\mathbf{r}, \omega)+S_{0}^{\min }(\mathbf{r}, \omega)}=C_{n}(\mathbf{r}, \omega),
$$

where $S_{n}^{\max }(\mathbf{r}, \omega)$ and $S_{n}^{\min }(\mathbf{r}, \omega)$ are the maximum and minimum values of the Stokes parameters measured around point $\mathrm{P}$, respectively, and $0 \leqslant C_{n}(\mathbf{r}, \omega) \leqslant 1$ [9]. The parameters $\mu_{n}\left(\mathbf{r}_{1}, \mathbf{r}_{2}, \omega\right)$ can be expressed as a normalized value of the generalized Stokes parameters [10],

$$
\mu_{n}\left(\mathbf{r}_{1}, \mathbf{r}_{2}, \omega\right)=\frac{S_{n}\left(\mathbf{r}_{1}, \mathbf{r}_{2}, \omega\right)}{\sqrt{\varphi_{0}\left(\mathbf{r}_{1}, \omega\right) \varphi_{0}\left(\mathbf{r}_{2}, \omega\right)}},
$$

where $\operatorname{tr} \overleftrightarrow{W}\left(\mathbf{r}_{i}, \mathbf{r}_{i}, \omega\right)=\varphi_{0}\left(\mathbf{r}_{i}, \omega\right)$, for $(i=1,2)$, are the spectral densities at slits $\rho_{1}$ and $\rho_{2}$, respectively (Fig. 1 ). When $S_{n}^{(1)}(\mathbf{r}, \omega) \approx S_{n}^{(2)}(\mathbf{r}, \omega)$, i.e., Stokes parameters at point $\mathrm{P}(\mathbf{r})$ due to both the beams being approximately same, using Eqs. (1) and (2), the real part of the phase term can be written in terms of the experimentally measurable quantities as

$$
\cos \beta_{n}=\frac{S_{n}(\mathbf{r}, \omega)-2 S_{n}^{(1)}(\mathbf{r}, \omega)}{2 S_{0}^{(1)}(\mathbf{r}, \omega) \times C_{n}(\mathbf{r}, \omega)} \quad \text { for }(n=0,1,2,3) .
$$

The imaginary part of the phase term can be obtained from Eq. (4) using simple trigonometric relation $\sin \beta_{n}=\sqrt{1-\cos ^{2} \beta_{n}}$. Using Eq. (3), we get

$$
\begin{aligned}
S_{n}\left(\mathbf{r}_{1}, \mathbf{r}_{2}, \omega\right)= & \left|\mu_{n}\left(\mathbf{r}_{1}, \mathbf{r}_{2}, \omega\right)\right|\left(\cos \beta_{n}+\mathrm{i} \sin \beta_{n}\right) \\
& \times \sqrt{\varphi_{0}\left(\mathbf{r}_{1}, \omega\right) \varphi_{0}\left(\mathbf{r}_{2}, \omega\right)} .
\end{aligned}
$$

Equation (5) with Eqs. (2) and (4) is used to determine the generalized Stokes parameters from the measured values of the usual Stokes parameters and the contrast parameters.

The experimental setup consisted of a randomly polarized He-Ne laser (Melles Griot). The laser beam with peak wavelength $632.8 \mathrm{~nm}$ was passed through a spatial-filter beam expander assembly, as shown in Fig. 1. The expanded beam was made incident on a double slit of a slit width of $150 \mu \mathrm{m}$ and a slit separation of $200 \mu \mathrm{m}$. The two beams emerging from the double slit interfered, and interference fringes were obtained in plane $\mathrm{R}$ at a distance $150 \mathrm{~cm}$ from the slit. The interference fringes are shown in Fig. 2(a). The spectral measurements were taken at and around an off-axis point $\mathrm{P}(\mathbf{r})$ using a fiber-coupled spectrometer (Photon Control, SPM002) interfaced with a personal computer (Fig. 1). The fringe pattern across the observation plane was scanned mounting the fiber tip on a micropositioner (Standa). The spectral profile of the laser beam at point $\mathrm{P}$ is shown in Fig. 2(b).

To determine the usual Stokes parameters due to single beam, one of the slits was closed using a stopper $\left(\mathrm{T}_{1}\right.$ or $\left.\mathrm{T}_{2}\right)$ and a combination of a linear polarizer and a quarter-wave plate was introduced in the beam path (Fig. 1). The four usual Stokes parameters due to the single beam were obtained by taking spectral measurements at point $\mathrm{P}$ using the following expressions:

$$
\begin{aligned}
& S_{0}^{1}(\mathbf{r}, \omega)=\varphi\left(0^{\circ}, 0^{\circ}\right)+\varphi\left(90^{\circ}, 0^{\circ}\right), \\
& S_{1}^{1}(\mathbf{r}, \omega)=\varphi\left(0^{\circ}, 0^{\circ}\right)-\varphi\left(90^{\circ}, 0^{\circ}\right), \\
& S_{2}^{1}(\mathbf{r}, \omega)=\varphi\left(45^{\circ}, 0^{\circ}\right)-\varphi\left(135^{\circ}, 0^{\circ}\right), \\
& S_{3}^{1}(\mathbf{r}, \omega)=\varphi\left(45^{\circ}, 45^{\circ}\right)-\varphi\left(135^{\circ}, 45^{\circ}\right) .
\end{aligned}
$$

Here $\varphi(\theta, \phi)$ is the spectral density at point $\mathrm{P}$ when the polarizer makes angle $\theta$ with the $x$ axis and $\phi$ is the orientation of optic axis of the quarter-wave plate with the incident polarization of light (the axis of the polarizer). The Stokes parameters due to the other beam were also determined at point $\mathrm{P}$ using the experimental data in Eqs. (6a)-(6d) and were found to be approximately same as they were for the first beam, i.e., $S_{n}^{(1)}(\mathbf{r}, \omega) \approx S_{n}^{(2)}(\mathbf{r}, \omega)$ for $(n=0,1,2,3)$. These values of the usual Stokes parameters at point $\mathrm{P}$ for a single beam are shown in Table 1 .

In a similar manner, using proper direction of polarization of the polarizers and appropriate orientation of the optic axis of the quarter-wave-plates placed in both the beams, the spectral measurements were made at point $\mathrm{P}$ for both the slits open simultaneously. With the help of Eq. (6), the usual Stokes pa-

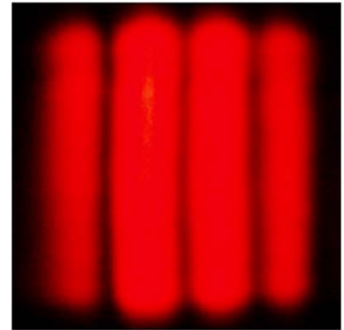

(a)

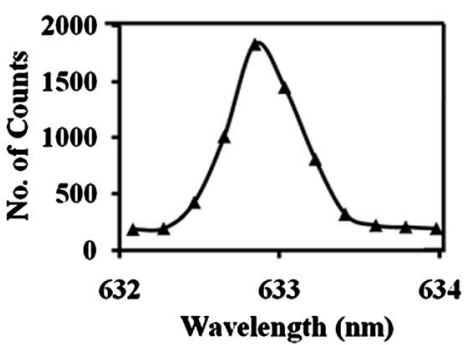

(b)
Fig. 2. (Color online) (a) Interference fringes due to the interferometer, obtained at the observation plane. (b) The spectrum of the laser beam (peak wavelength $=632.8 \mathrm{~nm}$ ) obtained at point $\mathrm{P}$ using the spectrometer. 
Table 1. Single Beam $S_{n}^{(1)}(\mathbf{r}, \omega)$ and Two Beam Usual

Stokes Parameters $S_{n}(r, \omega)($ for $n=0,1,2,3)$ Determined at Point $P$ in the Observation Plane

\begin{tabular}{cccc}
\hline$S_{0}^{(1)}(\mathbf{r}, \omega)$ & $1400 \pm 29.7$ & $S_{0}(\mathbf{r}, \omega)$ & $4000 \pm 53.1$ \\
$S_{1}^{(1)}(\mathbf{r}, \omega)$ & $30.5 \pm 2.5$ & $S_{1}(\mathbf{r}, \omega)$ & $82.5 \pm 8.4$ \\
$S_{2}^{(1)}(\mathbf{r}, \omega)$ & $21.5 \pm 5.3$ & $S_{2}(\mathbf{r}, \omega)$ & $80 \pm 9.2$ \\
$S_{3}^{(1)}(\mathbf{r}, \omega)$ & $157.3 \pm 9.3$ & $S_{3}(\mathbf{r}, \omega)$ & $822.5 \pm 22.3$ \\
\hline
\end{tabular}

rameters at the point due to both the beams were also determined. The values of these Stokes parameters are given in Table 1.

The contrast parameters were obtained using Eq. (2) by determining the maximum and minimum values of the Stokes parameters moving the fiber tip around point $\mathrm{P}$ on plane $\mathrm{R}$. The distance between the two consecutive positions of the fiber tip for maximum and minimum values of the contrast parameters was found to be $600 \mu \mathrm{m}$ approximately. The contrast parameters determined by visibility measurements are presented in Table 2. The phase part of the modulation parameters was determined using the aforementioned method, by putting the experimentally determined values of the usual Stokes parameters (Table 1) and the contrast parameters (Table 2) in Eq. (4). These values along with the experimentally obtained values of the spectral densities at the slits (without polarizers) were put in Eq. (5) giving the theoretically expected values of the generalized Stokes parameters. These complex two-point Stokes parameters are shown in Table 2. The uncertainties in the measurements calculated by repeating the experiments four times and shown with the experimental results in Tables 1 and 2 validate the experimental results to a large extent.

From Table 1 the polarization properties at point $\mathrm{P}$ due to a single beam and two beams could be analyzed. We see that the linear polarization along the $x$ axis, at $+45^{\circ}$ with the $x$ axis and the right circular polarization, are stronger than their counterparts, i.e., the $y$ axis; $-45^{\circ}$ and left circular polarization, respectively. It is observed that the two-beam usual Stokes parameters are different from the sum of the usual Stokes parameters for the individual beams. The difference occurs owing to an extra term called the modulation parameter $\mu_{n}\left(\mathbf{r}_{1}, \mathbf{r}_{2}, \omega\right)$ for the usual Stokes parameters. The contrast parameters in Table 2 show the absolute values of the modulation param-

Table 2. Contrast Parameters $C_{n}(r, \omega)$ and the Generalized Stokes Parameters $S_{n}\left(\mathbf{r}_{1}, \mathbf{r}_{2}, \omega\right)$ (for $n=0,1,2,3$ ) Determined for the Expanded Laser Beam

\begin{tabular}{lllc}
\hline$C_{0}(\mathbf{r}, \omega)$ & $0.79 \pm 0.08$ & $S_{0}\left(\mathbf{r}_{1}, \mathbf{r}_{2}, \omega\right)$ & $(600 \pm 19.2)+(930 \pm 26.0) \mathrm{i}$ \\
$C_{1}(\mathbf{r}, \omega)$ & $0.14 \pm 0.02$ & $S_{1}\left(\mathbf{r}_{1}, \mathbf{r}_{2}, \omega\right)$ & $(11 \pm 0.55)+(195 \pm 10.6) \mathrm{i}$ \\
$C_{2}(\mathbf{r}, \omega)$ & $0.15 \pm 0.04$ & $S_{2}\left(\mathbf{r}_{1}, \mathbf{r}_{2}, \omega\right)$ & $(19 \pm 0.87)+(207 \pm 12.1) \mathrm{i}$ \\
$C_{3}(\mathbf{r}, \omega)$ & $0.20 \pm 0.07$ & $S_{3}\left(\mathbf{r}_{1}, \mathbf{r}_{2}, \omega\right)$ & $(257 \pm 12.7)+(97 \pm 9.6) \mathrm{i}$ \\
\hline
\end{tabular}

eters. The real part of the generalized Stokes parameters shows a little dominance of the $x$ polarization of the beam over its $y$ polarization (Table 2 ). A comparison of the present results with those reported in Fig. 3 of [12] provides validity to the present experimental method.

In special circumstances, for axial point, the fringe intensity is found maximum, i.e., $\beta=0$. Thus we have $\mu_{n}\left(\mathbf{r}_{1}, \mathbf{r}_{2}, \omega\right)=\left|\mu_{n}\left(\mathbf{r}_{1}, \mathbf{r}_{2}, \omega\right)\right|$. Hence, Eq. (1) gives

$$
\mu_{n}\left(\mathbf{r}_{1}, \mathbf{r}_{2}, \omega\right)=\left|\mu_{n}\left(\mathbf{r}_{1}, \mathbf{r}_{2}, \omega\right)\right|=\frac{S_{n}(\mathbf{r}, \omega)-2 S_{n}^{(1)}(\mathbf{r}, \omega)}{2 S_{0}^{(1)}(\mathbf{r}, \omega)} .
$$

Thus using Eq. (7), the modulation parameters could be determined from the usual Stokes parameters alone. These values along with Eq. (2) could give the generalized Stokes parameters directly from the usual Stokes parameters.

In conclusion, the complex generalized Stokes parameters characterizing the polarization state of electromagnetic beams are determined experimentally for a pair of points in the cross section of the laser beam using the one-point usual Stokes parameters and the contrast parameters. The theoretical treatment presented using the electromagnetic spectral interference law is an outcome of the coherence theory in frequency domain. The present method finds an edge over the existing methods in that the change in polarization properties of the electromagnetic beams due to propagation can be obtained by simply determining the single-point polarization properties and the polarization visibility at a desired plane. The proposed method might have significant applications in polarization metrology.

The authors thank the Director, National Physical Laboratory, New Delhi 110012, India for permission to publish the paper. B. Kanseri acknowledges the Council of Scientific and Industrial Research, India for financial support as Senior Research Fellowship.

\section{References}

1. J. Ellis and A. Dogariu, Opt. Lett. 29, 536 (2004).

2. L. Mandel and E. Wolf, Optical Coherence and Quantum Optics (Cambridge U. Press, 1995).

3. G. G. Stokes, Trans. Cambridge Philos. Soc. 9, 399 (1852).

4. O. Korotkova and E. Wolf, Opt. Lett. 30, 198 (2005).

5. D. F. V. James, J. Opt. Soc. Am. A 11, 1641 (1994).

6. E. Wolf, Phys. Lett. A 312, 263 (2003).

7. E. Wolf, Opt. Lett. 28, 1078 (2003).

8. E. Wolf, Introduction to Theory of Coherence and Polarization of Light (Cambridge U. Press, 2007).

9. T. Setala, J. Tervo, and A. T. Friberg, Opt. Lett. 31, 2208 (2006).

10. T. Setala, J. Tervo, and A. T. Friberg, Opt. Lett. 31, 2669 (2006).

11. O. Korotkova, Opt. Lett. 261, 218 (2006).

12. B. Kanseri and H. C. Kandpal, Opt. Lett. 33, 2410 (2008) 